\title{
Exploring the value of Mycobacterium tuberculosis modified lipoprotein as a potential biomarker for TB detection in children
}

\author{
Xinxin Yang ${ }^{1,2}$, Matthew F. Wipperman ${ }^{1,3}$, Sharon Nachman ${ }^{4}$ (D) and Nicole S. Sampson ${ }^{1 *}$ (D)
}

\begin{abstract}
Background: Current TB diagnostic methods available have been developed for adults and development efforts have neglected the differences in disease and sampling that occur between adults and children. Diagnostic challenges are even greater in HIV co-infected children and infants.

Methods and results: We established a sandwich ELISA assay to detect Mycobacterium tuberculosis modified lipoprotein (TLP) ex vivo in plasma. The study population contains plasma samples from 21 patients with active TB and 24 control samples with no TB, collected in the International Maternal Pediatric Adolescent AIDS Clinical Trails (IMPAACT) P1041 study. Retrospective analysis was performed and the results demonstrate that the median plasma levels of TLP in control subjects are 2.7 fold higher than the median plasma values in active TB subjects $(p<0.001)$.
\end{abstract}

Conclusions: Plasma levels of TLP are elevated with active TB disease in HIV positive subjects and deserves further exploration as an indicator for TB detection in children.

Keywords: Childhood TB, HIV co-infection, Diagnosis, Sandwich ELISA, Lipoprotein, Low-density lipoprotein, apoB, Biomarker

\section{Background}

Childhood TB is estimated to contribute $12 \%$ of the disease burden, with approximately 10 million cases in 2019 worldwide [1]. Existing gold-standard diagnostic culture tools fail to confirm TB in most children, who typically have low bacterial counts and cannot produce sputum like adults. Diagnostic challenges are great in HIV co-infected children and infants [2], where the clinical presentation of pulmonary TB may be non-specific [3], acute [4, 5], and chest radiographs may be atypical [2]. Although rates of bacteriological confirmation appear similar in HIV-infected and uninfected children [6], diagnostic delay and HIV-related immune pathology

\footnotetext{
*Correspondence: nicole.sampson@stonybrook.edu

1 Department of Chemistry, Stony Brook University, Stony Brook, New York, NY 11794-3400, USA

Full list of author information is available at the end of the article
}

contribute to higher rates of $\mathrm{TB}$ disease progression with increased disease severity, morbidity, and mortality [7-10]. Infants, young children, and HIV-infected children are at increased risk of developing TB following infection and of disseminated or severe disease, including TB meningitis [11]. Because TB in children is typically paucibacillary, even if a sputum sample or gastric aspirate is obtained, the utility of PCR based diagnostic methods like GeneXpert is limited. More sensitive and childfriendly diagnostic tools are urgently needed to diagnose $\mathrm{TB}$ in children [12].

Mycobacterium tuberculosis $(M t b)$ is an intracellular pathogen that is capable of surviving and replicating within macrophages, the frontline of the innate host defense, and has evolved multiple mechanisms to escape these immune cells [13]. One of the immune evasion strategies of $M t b$ is the deregulation of lipid metabolism, leading to the formation of foamy macrophages (FM), 
a hallmark of granulomata in tuberculosis lesions [14]. However, only pathogenic mycobacterial strains including M. tuberculosis [15], M. avium, M. abscessus, or $M$. bovis, but not non-pathogenic mycobacterial strains, like M. smegmatis, induce foam cell formation upon infection $[16,17]$. Foam cells are associated with chronic inflammation in many metabolic diseases and certain cancers besides infectious diseases [18, 19]. For example, foamy macrophages are critical to the initial formation, development, and instability of atherosclerotic plaques and are therefore therapeutic targets in atherosclerosis. Foam cell formation is induced primarily by malondialdehydemodified low density lipoprotein (MDA-LDL) and not by native or extensively oxidized LDL in atherosclerosis [20]. Elevated plasma levels of MDA-LDL in patients are associated with acute coronary syndromes and are used as diagnostic tools clinically [21]. In contrast, the detailed mechanism of FM formation in $M t b$ is unknown. The current state of knowledge was that $M t b$ infection and $M t b$ trehalose dimycolate can induce FM formation [22]. Inspired by the association of MDA-LDL with FM and therefore atherosclerosis, we asked whether the modification of lipoprotein by $M t b$ may contribute the formation of FM and relate to the disease status of TB.

In this study we identified human lipoprotein modification specific to exposure to $M t b$. The pathogen modified host lipoprotein, TLP, is detectable ex vivo in plasma using a sandwich ELISA assay. We further evaluated the association between the presence of TLP and TB disease status in children.

\section{Methods}

1) Establish that TLP is 'real' and can be measured Preparation of malondialdehyde-conjugated LDL (MDA-LDL) [20] and Mtb-modified lipoprotein (TLP)

HepG2 human liver cells (ATCC HB-8065) were grown to $80 \%$ confluence in HepG2 growth media (DMEM, $10 \%$ fetal bovine serum, $20 \mathrm{mM} \mathrm{L}$-glutamine, $100 \mathrm{U} / \mathrm{mL}$ penicillin, $100 \mu \mathrm{g} / \mathrm{mL}$ streptomycin, and $10 \mathrm{mM}$ HEPES). Cells were grown for 4-5 days and culture supernatants were then harvested and concentrated. LDL particles were separated by density gradient ultracentrifugation and desalted by ultrafiltration through a $100-\mathrm{kDa}$ molecular weight filter. MDA-LDL was prepared by incubation $2 \mathrm{mg}$ protein $/ \mathrm{mL}$ of LDL with $2 \mu \mathrm{M}$ acrolein at $37{ }^{\circ} \mathrm{C}$ for $24 \mathrm{~h}$ under nitrogen atmosphere. Mycobacterium tuberculosis was cultured in Middlebrook 7H9 (broth) supplemented with $0.2 \%$ glycerol, $0.5 \%$ BSA, $0.08 \% \mathrm{NaCl}, 0.05 \%$ (v/v) tyloxapol to OD $\sim 0.7$. LDL was added and the culture was incubated at $37{ }^{\circ} \mathrm{C}$ for 7 days. TLP was isolated from the culture supernatant, concentrated and washed with PBS.

\section{Agarose gel electrophoresis and western blot analysis}

Agarose gel electrophoresis was performed in $0.06 \mathrm{M}$ barbital buffer $(\mathrm{pH}$ 8.6). The gel was $0.8 \%$ and stained with Sudan Red 7B. Lipoproteins were separated on a $6 \%$ sodium dodecyl sulfate polyacrylamide gel electrophoresis (SDS-PAGE), proteins were transferred to a PVDF membrane and immunoblotted with specific antibodies. The primary antibodies used for western blot were monoclonal anti-LDL (MDA oxidized) antibody (Abcam) and polyclonal anti-apoB Ab (H-300, Santa Cruz). Membranes were treated with anti-mouse, or anti-rabbit IgG HRP conjugates as secondary antibodies.

\section{2) Explore if induced TLP it potentially pathogenic by contributing to FM formation \\ THP-1 macrophage preparation and treatment}

THP-1 cells (ATCC TIB-202) were maintained in RPMI1640 medium containing heat-inactivated $10 \%$ fetal bovine serum (FBS), 0.05 mM 2-mercaptoethanol, $100 \mathrm{U} /$ $\mathrm{mL}$ penicillin and $100 \mathrm{mg} / \mathrm{mL}$ streptomycin (RPMI complete medium). The cells were plated into 6-well plates containing coverslips $\left(1.5 \times 10^{6} /\right.$ well $)$, treated with phorbol 12-myristate 13-acetate (PMA, $150 \mathrm{nM}$ ) in RPMI complete media. Media was replaced with fresh RPMI complete media at $48 \mathrm{~h}$ after plating. At $72 \mathrm{~h}$ after media change, THP-1 macrophages were treated with LDL, MDA-LDL and TLP at $200 \mu \mathrm{g} / \mathrm{mL}$ in PBS for $24 \mathrm{~h}$. All cells were incubated in a humid atmosphere at $37^{\circ} \mathrm{C}$ with $95 \%$ air and $5 \% \mathrm{CO}_{2}$.

\section{Lipid body staining and immunostaining}

Following lipoprotein treatment, macrophages were washed with PBS and fixed with $4 \%$ paraformaldehyde at room temperature for $30 \mathrm{~min}$. Cells were stained with Oil Red O solution for $20 \mathrm{~min}$ at room temperature. The slides were then counterstained with haematoxylin and observed under an inverted microscope (Zeiss Axiovert $200 \mathrm{M})$. Percent foam cell formation was quantified by counting stained versus total cells in 10 fields.

\section{3) Perform initial pilot evaluation of potential diagnostic value in a HIV + infant cohort Generation of anti-TLP antibodies [23, 24]}

Monoclonal antibodies against TLP were identified utilizing Human Combinatorial Antibody Library (HuCAL, Bio-Rad AbD Serotec GmbH). The HuCAL phage display library was depleted of antibodies that recognize intact LDL. The depleted library was panned for three rounds of binding, elution, and amplification to isolate antibodies specific for TLP. Binding of antiTLP antibodies to control antigens including BSA, HSA, N1-CD33-His6 and intact LDL, was checked by 
indirect ELISA assays, with anti-TLP antibodies as primary antibodies and an anti-Fab-AP conjugate (BioRad) as a secondary antibody.

\section{Sandwich ELISA assay}

Briefly, 96 well plates were coated overnight at $4{ }^{\circ} \mathrm{C}$ with $5 \mu \mathrm{g} / \mathrm{mL}$ capture antibody (AbD28580, amino acid sequence shown in Additional file 1: Table S2) in phosphate buffered saline (PBS). Plates were washed with PBS containing 0.05\% Tween-20 (PBST) 3 times, blocked with 3\% BSA in PBST for $1 \mathrm{~h}$ at room temperature. Then serial dilutions of standards and test samples in HISPEC assay diluent (Bio-Rad) were loaded and allowed to react for $2 \mathrm{~h}$ at room temperature. Plates were then washed 3 times with PBST and treated with $2 \mu \mathrm{g} / \mathrm{mL}$ of HRP conjugated detection antibody (AbD28582) in HISPEC assay diluent for $1 \mathrm{~h}$ at room temperature. After washing 6 times with PBST, the plates were developed with QuantaBlu fluorogenic peroxidase substrate kit (Thermo Scientific) for $30 \mathrm{~min}$ at room temperature. Fluorescence was recorded (ex. $320 \pm 25 \mathrm{~nm}$, em. $430 \pm 35 \mathrm{~nm}$ ).

\section{Subjects and samples}

Retrospective analysis was performed on a total of 45 plasma samples from International Maternal Pediatric Adolescent AIDS Clinical Trails (IMPAACT) P1041 [25, 26]. P1041 was a Phase II/ III, randomized, double-blind, placebo-controlled clinical trial to evaluate the efficacy of isoniazid prophylaxis on TB disease and latent $M t b$ infection free survival in HIV-infected and HIV-exposed, but uninfected infants up to 192 weeks of follow up [26, 27]. Samples include 24 with noTB and 21 with TBDIS, all HIV infected. Patients without $M t b$ infection were categorized as noTB; patients with a positive tuberculin skin test but lacking any clinical, radiographic or laboratory evidence of disease caused by $M t b$ were categorized as latent TB infection (LTBI); and patients presenting clinical, radiographic or laboratory evidence of disease caused by $M t b$ were categorized as TBDIS. Plasma samples were collected, frozen and thawed before use.

\section{Statistical analysis}

Categorical variables were compared using t-student test, whenever appropriate. Non-parametric tests (MannWhitney) were used for non-normally distributed variables. Assay accuracy, including 95\% confidence intervals, was assessed using sensitivity, specificity, predictive values and area under the ROC in the TB and non-TB groups. Statistical calculations were performed with GraphPad Prism ${ }^{\circledR}$ Software.

\section{Results}

1) Establish that TLP is distinct from known physiological modifications of LDL

Mtb modified lipoprotein (TLP) is different from MDA-LDL

We purified and treated native LDL with Mycobacterium tuberculosis in vitro to obtain $M t \mathrm{~b}$ modified lipoprotein (TLP). Decreased mobility of TLP in agarose electrophoresis indicates that TLP is more positively charged than native LDL (Fig. 1A and Additional file 1: Fig. S1A). In contrast, MDA-LDL, one of the end products of lipid peroxidation, is more negatively charged than native LDL [20]. The increased size of TLP compared to native LDL was also confirmed by dynamic light scattering analysis (Additional file 1: Table S1). Furthermore, TLP is recognized by anti-apolipoprotein $\mathrm{B}$ (apoB) antibodies but not anti-MDA-LDL antibodies, indicating TLP does not contain a malondialdehyde-modified apoB derivative (Fig. 1B and Additional file 1: Fig. S1B). We demonstrate that LDL is altered in the presence of $M t b$, and the resulting TLP is distinct from the typical atherosclerotic species malondialdehyde-modified LDL (MDA-LDL) present in human blood [28].

\section{2) Explore if induced TLP is potentially pathogenic by contributing to FM formation Mtb modified LDL induces lipid body accumulation in macrophages}

The induction of foamy macrophages have been reported as hallmarks in many pathologies associated with chronic proinflammatory stimuli including atherosclerosis [15]. It has also been reported that $M t b$ lipids induce the formation of giant multinuclear macrophages [29]. We therefore used foamy macrophage formation as a tool to assess the bioactive components that trigger a tissue response similar to granuloma formation in TB. Lipid bodies were stained with Oil red O. The buffer control showed no accumulation of lipid bodies and the cells remained rounded (Fig. 2A). LDL treated macrophage showed some lipid accumulation, with a commensurate increase in cell size (Fig. 2B). In contrast, MDA-LDL, commonly associated with atherosclerosis, treated macrophages exhibited more extensive accumulation of lipid bodies than the LDL treated macrophages. THP-1 macrophages treated with PMA do not entirely reproduce the response spectrum of primary monocyte-derived macrophages to activating stimuli [30]. However, foamy macrophage formation is a straightforward biological process and we used this simplified model for comparison to previous studies. These macrophage phenotypes are consistent with previous reports of in vitro foamy macrophage formation 


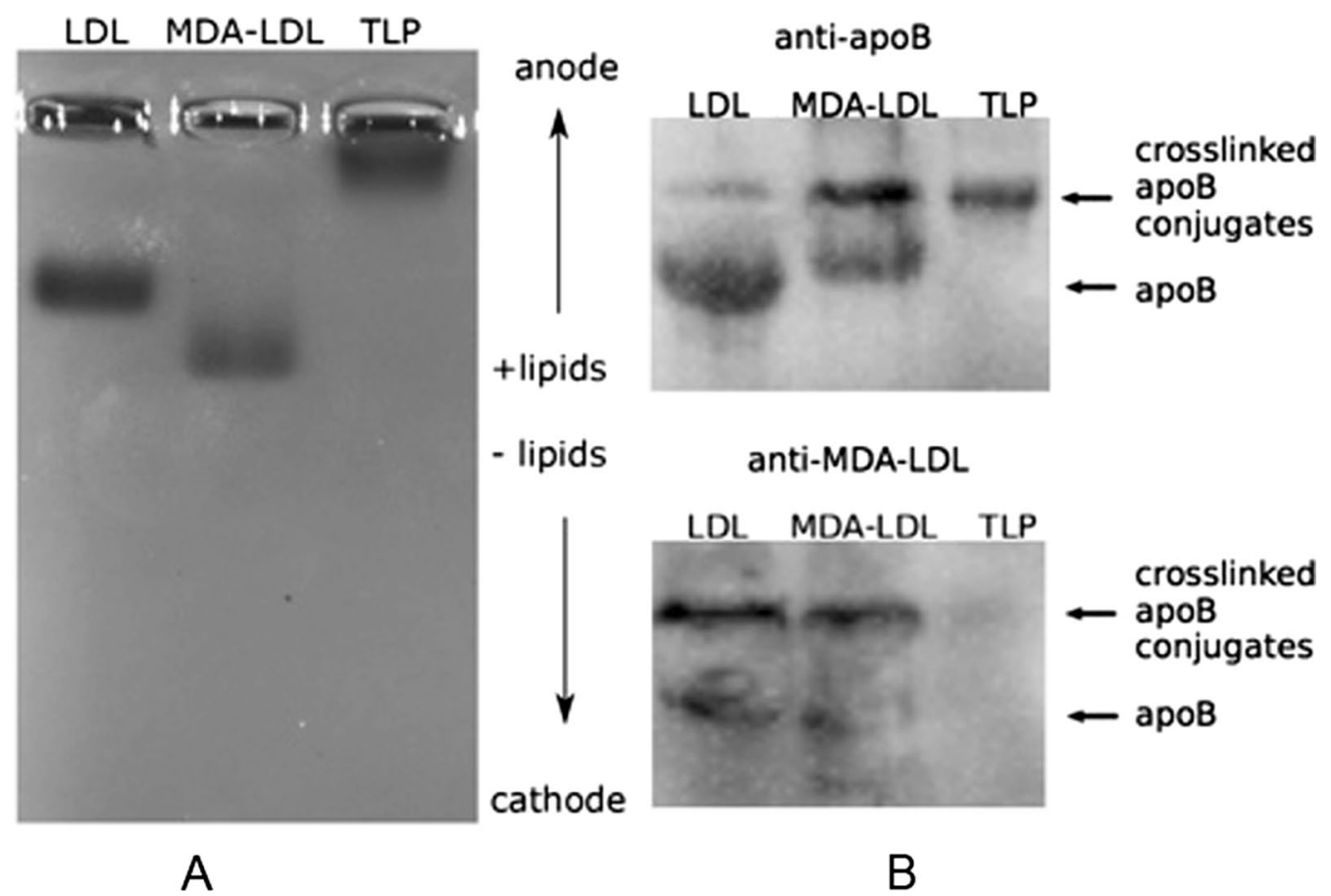

Fig. 1 TLP is different from MDA-LDL. A an agarose gel electrophoresis showing TLP is larger in size and more positively charged than native LDL. B Immunoblots showing TLP is recognized by anti- apolipoprotein B (apoB) antibodies (top) but not anti- MDA-LDL antibodies (bottom). Images of the entire gel and blots are in Additional file 1: Figure S1

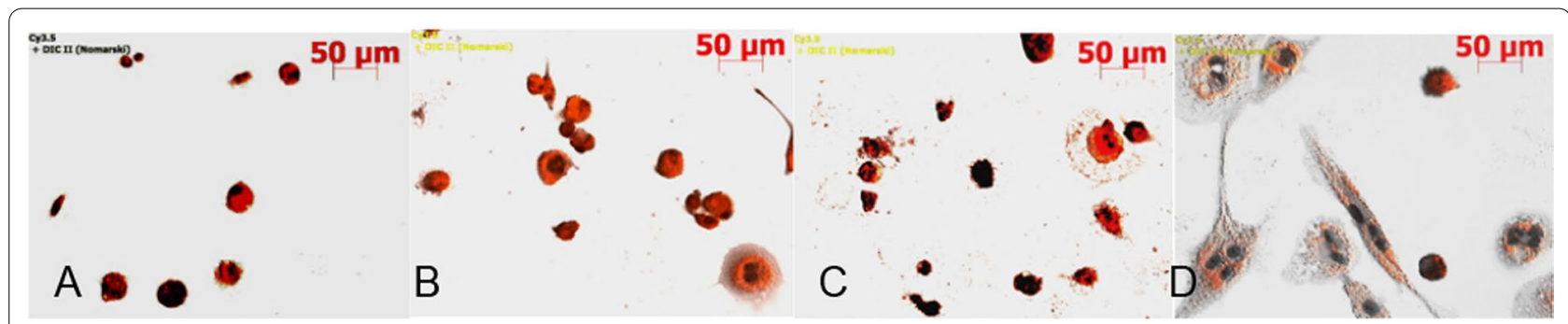

Fig. 2 TLP induces lipid body accumulation in macrophages. Microscopic images of Oil Red O-hematoxylin stained THP-1 macrophage culture treated with PBS control (A), LDL (B), MDA-LDL (C) and TLP (D) at $200 \mu \mathrm{g} / \mathrm{mL}$ for 2 days

[20, 31]. Most importantly, TLP treated macrophages had extensive lipid body accumulation per cell compared to macrophages treated with other LDLs (Fig. 2 and Additional file 1: Fig. S2B). Moreover, the TLPtreated macrophage morphology was distinct from other samples. The majority of the TLP-treated macrophages became multinucleated (Fig. 2D and Additional file 1: Fig. S2A) and developed long filopodia consistent with cell migration or exocytosis. Thus, we hypothesize that the modified LDL is detectable in vivo to serve as a biomarker for TB disease, and an antibody specific for recognition of TLP is an $M t b$-specific indicator of infection and progression to (active) TB disease.

\section{3) Perform initial pilot evaluation of potential diagnostic value of TLP in a HIV + infant cohort Anti-TLP monoclonal antibodies were generated and a TLP Sandwich ELISA assay was established}

We generated 13 anti-TLP monoclonal antibodies (FadA-FH, bivalent Fab-bacterial alkaline phosphatase fusion antibody followed by FLAG ${ }^{\circledR}$ and His6-tag) from the Human Combinatorial Antibody Library (HuCAL, BioRad AbD Serotec $\mathrm{GmbH}$ ) using in vitro selection and counter selection $[23,24]$. HuCAL is a phage display 
library containing highly specific, fully human monoclonal antibodies, with DNA sequence encoded for each antibody fragment. The HuCAL phage display library was depleted of antibodies that recognize LDL (Additional file 1: Figure S3). The depleted library was panned for three rounds of binding, elution, and amplification to isolate antibodies specific for TLP. Among the antibody hits selected, 13 antibodies were identified as unique by DNA sequencing. These antibodies were produced heterologously for further development. The cross-reactivity with native LDL, human serum albumin, bovine serum albumin, and $\mathrm{His}_{6}$ affinity tag was determined in an indirect ELISA format. Eight antibodies showed a specific signal for TLP at least fourfold above native LDL background (Additional file 1: Figure S3). We tested pairwise combinations of antibodies to identify antibody pairs that have non-overlapping epitopes suitable for use in a sandwich ELISA (Additional file 1: Figure S4A). 5 sandwich pairs were identified with good signal over background and the assay conditions were optimized for the best pair, AbD28582 and AbD28580 (amino acid sequence shown in Additional file 1: Table S2). We established the linear response of the sandwich ELISA (Additional file 1: Figure S4B) to antigen spiked into pediatric plasma. TLP is specifically detected at $>=4 \mathrm{ppm}$ in the presence of plasma LDL, which is around $10^{6} \mathrm{ng} / \mathrm{mL}$ [32].

\section{Levels of TLP are increased in patients with active TB compared to no TB controls}

We assayed stored plasma samples from the HIV IMPAACT P1041 trial for the presence of TLP. The median plasma levels of TLP was $494 \mathrm{ng} / \mathrm{mL}$ in control subjects, and 2.7 fold higher in active TB subjects (Fig. 3A). The difference between control subjects and active TB subjects is significant $(\mathrm{p}<0.001)$. The receiver operating characteristic (ROC) analysis describes the relationship between the sensitivity and specificity at any cut-off values. An ROC curve of TLP concentration for active TB versus control subjects is presented in Fig. 3B. The area under the ROC curve (AUC) is $0.86(95 \% \mathrm{CI}$ 0.75-0.97, $\mathrm{p}<0.0001)$. An optimal cutoff of $1064 \mathrm{ng} / \mathrm{mL}$ rendered $71 \%$ sensitivity and $88 \%$ specificity. Alternatively, a cutoff of $1230 \mathrm{ng} / \mathrm{mL}$ provided $57 \%$ sensitivity and $96 \%$ specificity.

We also monitored apoB levels, i.e., LDL levels, of all the samples using Human apoB ELISA kits (ab190806, Abcam) and found there was no linear correlation between TLP and apoB levels (Additional file 1: Figure S6A), suggesting that plasma LDL fluctuation does not interfere with TLP detection. There is a weak correlation between TLP levels and time before or after diagnosis (Additional file 1: Figure S6B). The samples collected before diagnosis may represent disease progression from no infection or infection but without clinical signs of disease to outward manifestation of disease. Samples collected after diagnosis may represent a mixed outcome of disease progression and anti- tubercular treatment. We do not have additional sample sets to demonstrate if anti-tubercular treatment affects TLP levels. However, the discrimination of TLP levels between control subjects and active TB subjects (post diagnosis with treatment less than 40 weeks) was improved with the
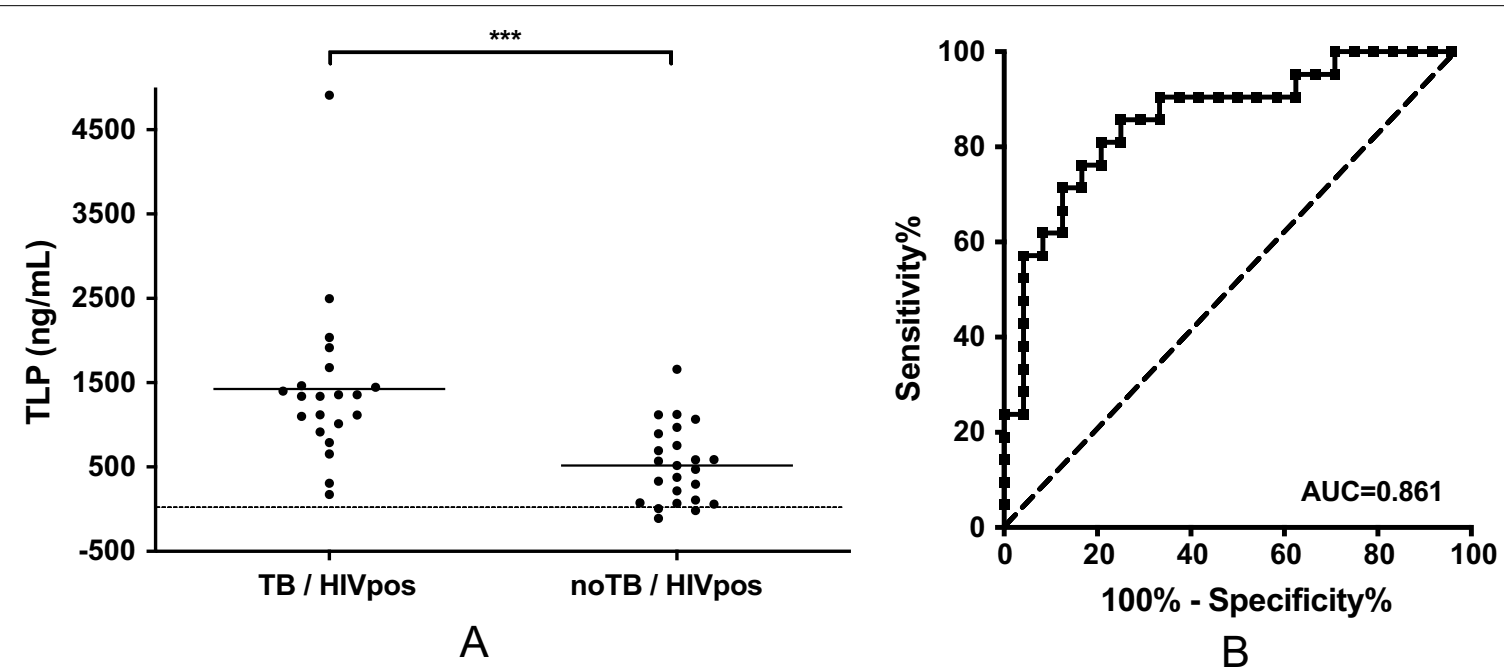

Fig. 3 Retrospective analysis of P1041 HIV positive plasma samples. A Plasma levels of TLP in HIV positive subjects clinically diagnosed probably having active stage tuberculosis disease (TB) and not having TB (noTB). Individual patients are depicted as dots with group mean. The dashed line represents the detection limit of the assay. ${ }^{* * *} p<0.001$. B ROC curve of TLP concentration for active TB versus control subjects of HIV positive patients. AUC, area under curve. The dashed line represents the no-discrimination line from the left bottom to the top right corners 
exclusion of samples collected after 40 weeks of treatment $(\mathrm{p}<0.0001)$, which may represent patients clear of TB (Additional file 1: Figure S6). ROC (Receiver operating characteristic) analysis provided an AUC (area under the ROC curve) of 0.88 (95\% CI 0.76-0.99, p<0.0001). An optimal cutoff of $632 \mathrm{ng} / \mathrm{mL}$ gave $67 \%$ sensitivity and $93 \%$ specificity. The results provide further evidence that TLP levels are associated with active TB disease. Overall, the results clearly highlight the potential of TLP as a biomarker in the diagnosis of active TB in HIV positive children.

\section{Discussion and conclusions}

The wide spectrum of disease observed in children, and the non-specific signs and symptoms especially in young and HIV + children, contribute to diagnostic delay and missed opportunities to detect TB, which in turn, favors TB disease progression and poor treatment outcomes. There is urgent need to search for a "Rapid Non-sputum-based Biomarker Test for Tuberculosis Detection" [12]. Most of the biomarkers currently studied are hostderived biomarkers including metabolites and cytokines $[33,34]$, the levels of which can be affected by many confounding factors. On the other hand, $M t b$ products can be detected directly in blood, sputum or urine, and are increasingly being used for diagnosis [35]. We have characterized a unique biomolecule, $M t b$ modified lipoprotein, which results from a combination of host and $M t b$ pathogen activity. Functionally, TLP increases the flux of lipid into activated macrophages, thereby stimulating their conversion into foamy multi-nucleated macrophages. The TLP-stimulated macrophages accumulate lipid bodies but have a distinct phenotype from MDALDL-stimulated macrophages (Fig. 2). We reason that detection of this biomolecule in patient plasma may withstand the heterogeneity associated with variations derived from host and be pathogen or disease specific. Thus, TLP detection may be used as TB diagnosis in young children.

The lipoprotein modification can take place in macrophages in contact with $M$. tuberculosis. There are also reports demonstrating that extracellular vesicles carrying bacterial components are released from infected macrophages and circulated beyond the site of infection [36], where host lipoproteins could be modified via contact with the extracellular vesicles. In addition, the intracellular bacilli are able to avoid killing by escaping phagosome-lysosome fusion. In certain individuals, especially children below 5 years of age and immunosuppressed subjects, the extracellular bacilli may disperse to distant metastatic sites via lymphatics and the bloodstream [37], where host lipoproteins may be modified by direct contact with M. tuberculosis. Our data demonstrate that the modified lipoprotein in the present study is distinct from other commonly recognized disease associated LDLs, which are typically negatively charged and smaller in particle size compared to native LDL [38].

The present study established a rapid, non-invasive, and robust sandwich ELISA assay for the detection of a novel biomolecule TLP in plasma. It would be beneficial to further refine the sensitivity and selectivity of the ELISA assay and determine the minimum required dilution to optimize the matrix effect, in order to standardize the test and use it as a diagnostic kit. We demonstrate that plasma levels of TLP are distinguishable in HIV positive subjects with active TB disease compared to no TB controls (Fig. 3). The elevation of plasma levels of TLP in HIV positive subjects with active TB disease suggests the detection of TLP is associated with TB active disease in HIV positive subjects. The AUC value of 0.86 evidenced the potential diagnostic value of TLP for TB detection in child. The target product profiles (TPP) adopted by the World Health Organization (WHO) in 2015 for a "Rapid Non-sputum-based Biomarker Test for Tuberculosis Detection" is a diagnostic sensitivity $\geq 66 \%$ for microbiologically confirmed pediatric TB and $98 \%$ specificity for a pediatric test [12]. The TPP proposed sensitivity is similar to the sensitivity of the Xpert MTB/RIF assay in microbiologically confirmed samples. Our assay renders $57 \%$ sensitivity and $96 \%$ specificity ( $21 \mathrm{~TB}$ and 24 control samples), which has the potential to meet the WHO TPP for detection of active TB in children, given the highly heterogenous population of P1041 samples, with respect to the bacteriological and clinical status.

Besides limited numbers of samples used in the current study, the heterogeneity of the clinical status of the samples may affect the accuracy of the test. Samples used in our study were obtained from IMPAACT P1041 clinical trial. The accuracy of TB diagnosis for the P1041 trial samples is not known due to the absence of good pediatric TB diagnostics. In addition, microbiological confirmation of TB disease was not obtained for any of these patients in the P1041 trial. TB disease subjects used in the present study are patients infected with TB presenting clinical symptoms of disease. The ELISA assays were performed retrospectively and the variance in storage conditions might affect the antigen integrity and therefore the accuracy of the assay. We have insufficient samples to include other clinical characteristics of the patients into data analysis. The clinical characteristics include but are not limited to BCG administration, immunosuppression status like CD4 counts, or clinical manifestation of tuberculosis (pulmonary and extra-pulmonary TB). The consideration of those characteristics may help define the limitation of our TLP assay or render improved accuracy with the combination of other clinical factors. 
We focused on HIV positive subjects in the current study. TB diagnosis is particularly difficult among HIV co-infected individuals who may have atypical, nonspecific clinical presentation, high rates of smear negative disease and high rates of extrapulmonary $\mathrm{TB}$ [39]. P1041 samples were obtained from TB endemic settings, therefore patients were typically being diagnosed at more advanced stages than those living in the US. Therefore, the heterogenous stages of TB disease may contribute to the wide dispersity of TLP levels in TB disease subjects (Fig. 3A), which is common among TB diagnostic assays. In addition, the duration of anti-tubercular treatment was not consistent among patients in the present study. Change of LDL modification is a dynamic process and may have the potential to monitor treatment outcome. Although not statistically significant, the slight decrease in the TLP level post diagnosis/ anti-tubercular treatment of 40 weeks may be related to a reduction in microbial load (Additional file 1: Figure S6A and B). Further studies are required to elucidate the effect of different disease status and/or treatment status on TLP levels in plasma, and to extend our preliminary results to other populations such as HIV negative subjects and adults. Although the accessibility and better-defined TB status of adults might be beneficial for feasibility testing, the matured immune system of adults might add complexity to interpretation of the assay.

It is a growing notion in the field that a single biomarker will not be sufficient for distinguishing TB status in different patient groups, and multiple biomarkers may be used to increase sensitivity and specificity. Further gains in clinical sensitivity and specificity may be obtained by combining the current assay with other biomarkers or diagnostic tests.

\section{Abbreviations \\ TB: Tuberculosis; Mtb: Mycobacterium tuberculosis; ELISA: Enzyme-linked immunosorbent assay; FM: Foamy macrophages; MDA-LDL: Malondialdehyde- modified low density lipoprotein; TLP: Mycobacterium tuberculosis Modified lipoprotein; ROC: Receiver operating characteristic; AUC: Area under the ROC curve.}

\section{Supplementary Information}

The online version contains supplementary material available at https://doi. org/10.1186/s12879-022-07140-9.

Additional file 1. Supplemental Information.

Acknowledgements

We thank Guannan Chen for help with initial LDL purification experiments.

\section{Authors' contributions}

SN, MFW, XY, NSS conceived the experiments. SN, MFW, obtained sample resource. $X Y$ performed the experiments. $X Y$, NSS wrote the main manuscript text. XY prepared all figures. All authors read and approved the final manuscript.

\section{Funding}

Research reported in this publication was supported by the National Heart, Lung, and Blood Institute of the National Institutes of Health under Award Number U01HL127522, and National Institute of Allergy and Infectious Diseases (NIAID) under award number R41Al136071 to Chronus Pharmaceuticals, Inc with a subcontract to Stony Brook University (NSS) and the National Center for Advancing Translational Sciences under award number TL1TR002386 (MFW). Overall support for the International Maternal Pediatric Adolescent AIDS Clinical Trials (IMPAACT) Network was provided by the National Institute of Allergy and Infectious Diseases (NIAID) of the National Institutes of Health (NIH) under Award Numbers UM1AI068632 (IMPAACT LOC), UM1AI068616 (IMPAACT SDMC) and UM1AI106716 (IMPAACT LC), with co-funding from the Eunice Kennedy Shriver National Institute of Child Health and Human Development (NICHD) and the National Institute of Mental Health (NIMH). The content is solely the responsibility of the authors and does not necessarily represent the official views of the $\mathrm{NIH}$.

\section{Availability of data and materials}

The datasets used and/or analysed during the current study are available from the corresponding author on reasonable request.

\section{Declarations}

\section{Ethics approval and consent to participate}

All methods were carried out in accordance with relevant guidelines and regulations. The NIH-IMPAACT Scientific Leadership Group (SLG) reviewed and approved the use of P1041 trial samples under NWCS127. The Wits Health Consortium, University of Witwatersrand, South Africa provided deidentified samples to Stony Brook University for which informed consent had been obtained from a parent and/or legal guardian of all participants. The Stony Brook University CORIHS determined the experiments (IRB\# 687246-1) in this manuscript were not human subjects research (exemption 4) under either United States Department of Health and Human Services 45 CFR part 46, subpart A, "the Common Rule" or United States Food and Drug Administration (FDA) Regulations 21 CFR parts 50, 56, 312 and 812.

\section{Consent for publication}

Not applicable.

\section{Competing interests}

NSS is President and co-owner of Chronus Pharmaceuticals, Inc. XY was an employee of both Chronus Pharmaceuticals, Inc and Stony Brook University. All intellectual property is currently owned by Stony Brook University. SN and MFW have no conflict of interest.

\section{Author details}

${ }^{1}$ Department of Chemistry, Stony Brook University, Stony Brook, New York, NY 11794-3400, USA. ${ }^{2}$ Chronus Pharmaceuticals, Inc., 25 Health Sciences Drive, Stony Brook, New York, NY 11790, USA. ${ }^{3}$ Present Address: Clinical and Translational Science Center, Weill Cornell Medicine, New York, NY 10065, USA. ${ }^{4}$ Department of Pediatrics, Renaissance School of Medicine, Stony Brook University, Stony Brook, New York, NY 11794-8434, USA.

Received: 2 April 2021 Accepted: 8 February 2022

Published online: 17 February 2022

\section{References}

1. Global tuberculosis report. https://apps.who.int/iris/bitstream/handle/ 10665/336069/9789240013131-eng.pdf.

2. Cotton MF, Schaaf HS, Hesseling AC, Madhi SA. HIV and childhood tuberculosis: the way forward. Int J Tuberculosis Lung Dis. 2004;8(5):675-82.

3. Marais BJ, Gie RP, Obihara CC, Hesseling AC, Schaaf HS, Beyers N. Well defined symptoms are of value in the diagnosis of childhood pulmonary tuberculosis. Arch Dis Child. 2005;90(11):1162-5. 
4. Moore DP, Klugman KP, Madhi SA. Role of Streptococcus pneumoniae in hospitalization for acute community-acquired pneumonia associated with culture-confirmed Mycobacterium tuberculosis in children: a pneumococcal conjugate vaccine probe study. Pediatr Infect Dis J. 2010;29(12):1099-1004.

5. Zar HJ, Tannenbaum E, Apolles P, Roux P, Hanslo D, Hussey G. Sputum induction for the diagnosis of pulmonary tuberculosis in infants and young children in an urban setting in South Africa. Arch Dis Child. 2000;82(4):305-8.

6. Walters E, Cotton MF, Rabie H, Schaaf HS, Walters LO, Marais BJ. Clinical presentation and outcome of tuberculosis in human immunodeficiency virus infected children on anti-retroviral therapy. BMC Pediatr. 2008;8:1.

7. Jeena PM, Mitha T, Bamber S, Wesley A, Coutsoudis A, Coovadia HM. Effects of the human immunodeficiency virus on tuberculosis in children. Tubercle Lung Dis. 1996;77(5):437-43.

8. Schaaf HS, Gie RP, Beyers N, Smuts N, Donald PR. Tuberculosis in infants less than 3 months of age. Arch Dis Child. 1993;69(3):371-4.

9. Jeena PM, Pillay P, Pillay T, Coovadia HM. Impact of HIV-1 co-infection on presentation and hospital-related mortality in children with culture proven pulmonary tuberculosis in Durban, South Africa. Int J Tubercul Lung Dis. 2002;6(8):672-8

10. Madhi SA, Huebner RE, Doedens L, Aduc T, Wesley D, Cooper PA. HIV-1 co-infection in children hospitalised with tuberculosis in South Africa. Int J Tubercul Lung Dis. 2000;4(5):448-54.

11. Marais BJ, Gie RP, Schaaf HS, Hesseling AC, Obihara CC, Starke JJ, Enarson DA, Donald PR, Beyers N. The natural history of childhood intra-thoracic tuberculosis: a critical review of literature from the pre-chemotherapy era. Int J Tubercul Lung Dis. 2004;8(4):392-402.

12. Denkinger CM, Kik SV, Cirillo DM, Casenghi M, Shinnick T, Weyer K, Gilpin C, Boehme CC, Schito M, Kimerling M, et al. Defining the needs for next generation assays for tuberculosis. J Infect Dis. 2015;211(Suppl 2):S29-38.

13. Abdalla AE, Ejaz H, Mahjoob MO, Alameen AAM, Abosalif KOA, Elamir MYM, Mousa MA. Intelligent mechanisms of macrophage apoptosis subversion by Mycobacterium. Pathogens. 2020;9(3):218.

14. Genoula M, Marin Franco JL, Dupont M, Kviatcovsky D, Milillo A, Schierloh P, Morana EJ, Poggi S, Palmero D, Mata-Espinosa D, et al. Formation of foamy macrophages by tuberculous pleural effusions is triggered by the interleukin-10/signal transducer and activator of transcription 3 axis through ACAT upregulation. Front Immunol. 2018;9:459.

15. Russell DG, Cardona PJ, Kim MJ, Allain S, Altare F. Foamy macrophages and the progression of the human tuberculosis granuloma. Nat Immunol. 2009;10(9):943-8

16. Viljoen A, Blaise M, de Chastellier C, Kremer L. MAB_3551c encodes the primary triacylglycerol synthase involved in lipid accumulation in Mycobacterium abscessus. Mol Microbiol. 2016;102(4):611-27.

17. Caire-Brandli I, Papadopoulos A, Malaga W, Marais D, Canaan S, Thilo L, de Chastellier C. Reversible lipid accumulation and associated division arrest of Mycobacterium avium in lipoprotein-induced foamy macrophages may resemble key events during latency and reactivation of tuberculosis. Infect Immun. 2014;82(2):476-90.

18. Guerrini V, Gennaro ML. Foam cells: one size doesn't fit all. Trends Immunol. 2019;40(12):1163-79.

19. Agarwal P, Combes TW, Shojaee-Moradie F, Fielding B, Gordon S, Mizrah $\checkmark$, Martinez FO. Foam cells control Mycobacterium tuberculosis Infection. Front Microbiol. 2020;11:1394.

20. Watanabe K, Nakazato Y, Saiki R, Igarashi K, Kitada M, Ishii I. Acroleinconjugated low-density lipoprotein induces macrophage foam cell formation. Atherosclerosis. 2013;227(1):51-7.

21. Holvoet P, Vanhaecke J, Janssens S, Van de Werf F, Collen D. Oxidized LDL and malondialdehyde-modified LDL in patients with acute coronary syndromes and stable coronary artery disease. Circulation. 1998;98(15):1487-94.

22. Geisel RE, Sakamoto K, Russell DG, Rhoades ER. In vivo activity of released cell wall lipids of Mycobacterium bovis bacillus Calmette-Guerin is due principally to trehalose mycolates. J Immunol. 2005;174(8):5007-15.

23. Knappik A, Ge L, Honegger A, Pack P, Fischer M, Wellnhofer G, Hoess A, Wolle J, Pluckthun A, Virnekas B. Fully synthetic human combinatorial antibody libraries (HuCAL) based on modular consensus frameworks and CDRs randomized with trinucleotides. J Mol Biol. 2000;296(1):57-86.

24. Prassler J, Thiel S, Pracht C, Polzer A, Peters S, Bauer M, Norenberg S, Stark Y, Kolln J, Popp A, et al. HuCAL PLATINUM, a synthetic Fab library optimized for sequence diversity and superior performance in mammalian expression systems. J Mol Biol. 2011;413(1):261-78.

25. Madhi SA, Nachman S, Violari A, Kim S, Cotton MF, Bobat R, Jean-Philippe P, McSherry G, Mitchell C, Team PS. Primary isoniazid prophylaxis against tuberculosis in HIV-exposed children. N Engl J Med. 2011;365(1):21-31.

26. A randomized, double blind, placebo controlled trial to determine the efficacy of isoniazid ( $\mathrm{INH})$ in preventing tuberculosis disease and latent tuberculosis infection among infants with perinatal exposure to HIV. https://impaactnetwork.org/DocFiles/P1041/P1041V2_11Jul07.pdf.

27. Gupta A, Montepiedra G, Gupte A, Zeldow B, Jubulis J, Detrick B, Violari A, Madhi S, Bobat R, Cotton M, et al. Low vitamin-D levels combined with PKP3-SIGIRR-TMEM16J host variants is associated with tuberculosis and death in HIV-infected and -exposed infants. PloS One. 2016;11(2):e0148649.

28. Yoshida M, Higashi K, Kobayashi E, Saeki N, Wakui K, Kusaka T, Takizawa H, Kashiwado K, Suzuki N, Fukuda K, et al. Correlation between images of silent brain infarction, carotid atherosclerosis and white matter hyperintensity, and plasma levels of acrolein, IL-6 and CRP. Atherosclerosis. 2010;211(2):475-9.

29. Puissegur MP, Lay G, Gilleron M, Botella L, Nigou J, Marrakchi H, Mari B, Duteyrat JL, Guerardel Y, Kremer L, et al. Mycobacterial lipomannan induces granuloma macrophage fusion via a TLR2-dependent, ADAM9and beta1 integrin-mediated pathway. J Immunol. 2007;178(5):3161-9.

30. Tedesco S, De Majo F, Kim J, Trenti A, Trevisi L, Fadini GP, Bolego C, Zandstra PW, Cignarella A, Vitiello L. Convenience versus biological significance: are PMA-differentiated THP-1 cells a reliable substitute for blood-derived macrophages when studying in vitro polarization? Front Pharmacol. 2018;9:71

31. Nezami N, Ghorbanihaghjo A, Rashtchizadeh N, Argani H, Tafrishinejad A, Ghorashi S, Hajhosseini B. Atherogenic changes of low-density lipoprotein susceptibility to oxidation, and antioxidant enzymes in pulmonary tuberculosis. Atherosclerosis. 2011;217(1):268-73.

32. Sacks FM, Moye LA, Davis BR, Cole TG, Rouleau JL, Nash DT, Pfeffer MA, Braunwald E. Relationship between plasma LDL concentrations during treatment with pravastatin and recurrent coronary events in the cholesterol and recurrent events trial. Circulation. 1998;97(15):1446-52.

33. Weiner J, Kaufmann SH. Recent advances towards tuberculosis control: vaccines and biomarkers. J Intern Med. 2014;275:467.

34. Walzl G, Ronacher K, Hanekom W, Scriba TJ, Zumla A. Immunological biomarkers of tuberculosis. Nat Rev Immunol. 2011;11(5):343-54.

35. Goletti D, Lee MR, Wang JY, Walter N, Ottenhoff THM. Update on tuberculosis biomarkers: from correlates of risk, to correlates of active disease and of cure from disease. Respirology. 2018;23(5):455-66.

36. Athman JJ, Wang Y, McDonald DJ, Boom WH, Harding CV, Wearsch PA. Bacterial membrane vesicles mediate the release of Mycobacterium tuberculosis lipoglycans and lipoproteins from infected macrophages. J Immunol. 2015;195(3):1044-53.

37. Grosset J. Mycobacterium tuberculosis in the extracellular compartment: an underestimated adversary. Antimicrob Agents Chemother. 2003;47(3):833-6.

38. Orekhov AN. LDL and foam cell formation as the basis of atherogenesis. Curr Opin Lipidol. 2018;29(4):279-84.

39. Scott $L$, da Silva P, Boehme CC, Stevens W, Gilpin CM. Diagnosis of opportunistic infections: HIV co-infections-tuberculosis. Curr Opin HIV AIDS. 2017;12(2):129-38.

\section{Publisher's Note}

Springer Nature remains neutral with regard to jurisdictional claims in published maps and institutional affiliations. 